\title{
Hypothermie thérapeutique préhospitalière après arrêt cardiaque : état des lieux
}

\author{
Prehospital-induced hypothermia after out-of-hospital cardiac arrest: a national survey in France
}

\author{
M. Tesnière $\cdot$ S. Baré $\cdot$ F. Vitrat $\cdot$ E. Haller $\cdot$ P. Usseglio
}

Reçu le 24 février 2011 ; accepté le 2 mai 2011

(C) SFMU et Springer-Verlag France 2011

L'hypothermie thérapeutique (HT) après arrêt cardiaque (AC) a montré des bénéfices en termes de pronostic neurologique et de mortalité $[1,2]$. Elle est indiquée entre 32 et $34{ }^{\circ} \mathrm{C}$ dans la prise en charge des patients toujours comateux après reprise d'une activité cardiaque spontanée (RACS) [3]. En France, la prise en charge médicale des AC extrahospitaliers est le plus souvent assurée par les structures mobiles d'urgence et de réanimation (Smur).

Nous avons conduit une enquête auprès de toutes les Smur de France métropolitaine, entre octobre et décembre 2009. Le questionnaire était soumis à un médecin thésé de chaque Smur. Le critère de jugement principal était l'existence d'un protocole de service pour la réalisation d'une HT préhospitalière dans les $\mathrm{AC}$ non traumatiques de l'adulte. Les 354 Smur contactées ont toutes répondu au questionnaire. Trente-neuf (11\%) disposaient d'un protocole d'HT. Parmi ces 39 Smur qui utilisaient un protocole : 32 (82\%) ne tenaient pas compte du rythme cardiaque initial et débutaient l'HT aussi bien pour les AC par FV/TV que pour les asystolies et les activités électriques sans pouls. Vingt-six (67\%) utilisaient une méthode d'HT par perfusion de liquide froid, six $(15 \%)$ utilisaient un refroidissement externe et sept (18\%) l'association des deux méthodes. En ce qui concerne les traitements médicamenteux, 26 Smur (67\%) utilisaient à la fois un curare et une sédation. Une sédation seule était prescrite par cinq Smur (13\%). Huit Smur (20\%) n'associaient aucun traitement médicamenteux à l'HT. Enfin, 15 Smur $(38 \%)$ monitoraient la température après mise en route de l'HT.

Cette enquête montre que peu de Smur réalisent une HT et que les procédures sont très différentes d'une structure à l'autre. L'HT reste un traitement majeur de la réanimation post-AC comme l'ont confirmé les dernières recommanda-

M. Tesnière $(\bowtie) \cdot S$. Baré $\cdot$ F. Vitrat $\cdot$ E. Haller $\cdot$ P. Usseglio Samu 73, centre hospitalier de Chambéry, BP 1125 ,

F-73011 Chambéry cedex, France

e-mail : tesniere.marc@yahoo.fr tions de l'European Resuscitation Council [3]. Les indications ont d'ailleurs été élargies aux AC intrahospitaliers, aux enfants et à tous les rythmes initiaux, même si le niveau de preuve est moindre [4]. Par contre, la question du délai optimal pour initier l'HT reste posée. Les données expérimentales suggèrent que les effets protecteurs de l'hypothermie sont d'autant plus marqués que le traitement est précoce, mais n'ont pas été confirmées en pratique clinique. Par ailleurs, les contraintes spécifiques à l'exercice préhospitalier peuvent limiter la réalisation de l'HT. L'application de draps mouillés ou de poches de glace et la perfusion de liquide froid sont les deux méthodes utilisées en préhospitalier. Cependant, toutes les Smur ne disposent pas d'un réfrigérateur embarqué dans leurs véhicules, ce qui constitue un facteur limitant.

Pour conclure, on peut dire que la question de l'initiation de l'HT en préhospitalier reste d'actualité. Une étude multicentrique randomisée française évaluant le pronostic neurologique du patient en fonction du délai de mise en route de l'HT (Smur versus hôpital) aurait un intérêt certain.

Conflit d'intérêt : les auteurs ne déclarent aucun conflit d'intérêt.

\section{Références}

1. Bernard SA, Gray TW, Buist MD, et al (2002) Treatment of comatose survivors of out-of-hospital cardiac arrest with induced hypothermia. N Engl J Med 346:557-63

2. Hypothermia after Cardiac Arrest Study Group (2002) Mild therapeutic hypothermia to improve the neurologic outcome after cardiac arrest. N Engl J Med 346:549-56

3. Deakin CD, Nolan JP, Soar J, et al (2010) European Resuscitation Council Guidelines for Resuscitation 2010 Section 4. Adult advanced life support. Resuscitation 81:1305-52

4. Dumas F, Grimaldi D, Zuber B, et al (2011) Is hypothermia after cardiac arrest effective in both shockable and nonshockable patients: insights from a large registry. Circulation 123:877-86 